\title{
Chronic Human Pegivirus 2 without Hepatitis C Virus Co-infection
}

\author{
Kelly E. Coller, Veronica Bruce, Michael Cassidy, Jeffrey Gersch, Matthew B. Frankel, Ana Vallari, \\ Gavin Cloherty, John Hackett, Jr., Jennifer L. Evans, Kimberly Page, George J. Dawson
}

\begin{abstract}
Most human pegivirus $2(\mathrm{HPgV}-2)$ infections are associated with past or current hepatitis $\mathrm{C}$ virus (HCV) infection. $\mathrm{HPgV}-2$ is thought to be a bloodborne virus: higher prevalence of active infection has been found in populations with a history of parenteral exposure to viruses. We evaluated longitudinally collected blood samples obtained from injection drug users (IDUs) for active and resolved HPgV-2 infections using a combination of HPgV-2specific molecular and serologic tests. We found evidence of HPgV-2 infection in $11.2 \%(22 / 197)$ of past or current HCV-infected IDUs, compared with $1.9 \%$ (4/205) of an HCV-negative IDU population. Testing of available longitudinal blood samples from HPgV-2-positive participants identified 5 with chronic infection ( $>6$ months viremia in $>3$ timepoints); 2 were identified among the HCVpositive IDUs and 3 among the HCV-negative IDUs. Our findings indicate that HPgV-2 can establish chronic infection and replicate in the absence of HCV.
\end{abstract}

The recently identified second human pegivirus 1 (HPgV-2 or HHpgV-1) is a bloodborne flavivirus: little is known about the potential clinical significance of infection $(1,2)$. Active or resolved HPgV-2 infection has been detected worldwide in cohorts associated with risk for parenteral exposure to bloodborne pathogens $(1,3-5)$. In a study in which hepatitis C virus (HCV) status was determined (3), $1.2 \%$ of $\mathrm{HCV}$ positive were actively infected with $\mathrm{HPgV}-2$; none of the 1,306 HCV-negative participants (volunteer blood donors, HBV infected, HIV infected) were actively infected. In another study, participants with concurrent $\mathrm{HIV} / \mathrm{HCV}$ infection or injection drug users (IDUs) had a higher prevalence $(3.0 \%-5.7 \%)$ of active $\mathrm{HPgV}-2$ infection (4-6). Although active HPgV-2 has been found in other populations (e.g., hemophiliacs or others,

\footnotetext{
Author affiliations: Abbott Laboratories, Abbott Park, Illinois, USA

(K.E. Coller, M. Cassidy, J. Gersch, M.B. Frankel, A. Vallari,

G. Cloherty, J. Hackett, Jr., G.J. Dawson); University of New

Mexico, Albuquerque, New Mexico, USA (V. Bruce, K. Page);

University of California San Francisco, San Francisco, California,

USA (J.L. Evans)
}

DOI: https://doi.org/10.3201/eid2602.190434 with risk for parenteral exposure), their HCV antibody status was not determined $(6,7)$.

Previous studies indicate that $\mathrm{HPgV}-2$ can establish a chronic infection characterized by detectable viremia for $>6$ months $(2,6)$. Most chronic HPgV-2 cases are associated with active HCV infection $(2,6)$. In chronic HPgV-2 cases in which HCV RNA has not been detected, the presence of HCV antibodies (indicating a resolved infection) was not determined; thus, it is unclear whether previous HCV infection played a role in the initial HPgV-2 infection. Despite observations of $\mathrm{HCV}$ and $\mathrm{HPgV}-2$ co-infection, no evidence has been reported that $\mathrm{HPgV}-2$ infection exacerbates HCV infection $(5,6,8)$ or that co-infection influences the replication of either virus.

We examined a cohort of IDUs for whom longitudinal samples were available. We monitored the cohort for HCV status by HCV antibodies, RNA, or both, with the intent of capturing nascent HCV infections (9). We performed initial testing for $\mathrm{HPgV}-2$ (RNA and antibodies) on baseline and last collected samples. We further characterized longitudinal samples from available participants that showed active or resolved $\mathrm{HPgV}-2$ infection upon testing initial or last timepoints. We hypothesized that the IDUs would have similar prevalence of $\mathrm{HPgV}-2$ as shown in a previous study of HCV-infected persons with unknown IDU status, and that by studying IDUs without HCV infection we would uncover HPgV-2 infection in the absence of HCV. Last, we hypothesized that longitudinal samples from IDUs would reveal whether $\mathrm{HPgV}-2$ can establish a persistent infection in the absence of HCV co-infection.

\section{Materials and Methods}

\section{Samples}

We obtained samples from the U-Find-Out (UFO) Study, an ongoing prospective observational study of young adult active injectors, $<30$ years of age at enrollment, that was initiated in 2003 in the San Francisco Bay area (California, USA). Details of enrollment 
methods and follow-up have been described previously $(9,10)$. In brief, young adult IDUs were recruited from neighborhoods where IDUs were known to congregate and invited to participate in a field study for eligibility screening. Eligible persons were those who reported injection drug use in the prior 30 days, were $<30$ years of age, spoke English, had no plans to travel outside of the San Francisco Bay area for $>3$ months, and had negative or unknown HCV status. $\mathrm{HCV}$ antibody-positive persons were admitted into the study if their HCV RNA status was negative or unknown; those identified as HCV infected (RNA positive) at baseline were not enrolled into follow-up.

Eligible consenting participants were asked to complete a baseline interviewer-administered structured questionnaire that queried sociodemographics, parenteral and sexual risk behaviors and exposures, injecting exposures (e.g., frequency of injecting, number of persons injected with, types of drugs injected), alcohol use, and prevention and health service use. They were also asked to provide blood samples for $\mathrm{HCV}$ testing, including both $\mathrm{HCV}$ antibodies and HCV RNA, and for storage. Before 2012, all participants provided samples for $\mathrm{HCV}$ antibodies (using standard laboratory-based testing) and for a qualitative HCV RNA status determination using a nucleic acid amplification test (Procliex HIV-1/HCV assay; Gen-Probe Inc., https://www.novartis.com). Beginning in May 2012, HCV antibody testing was primarily conducted using a rapid test (OraSure Technologies, https://www.orasure.com) by fingerstick capillary blood collection; however, venipuncture was still used to collect specimens for RNA testing and sample storage. Baseline samples on 402 participants were selected as the initial sample set (Figure). A total of $205(51.0 \%)$ samples were negative and 197 (49.0\%) positive for HCV antibodies and HCV RNA at baseline. HCV-positive in this current study is defined as any evidence of HCV infection (RNA or HCV antibodies), past or present; HCV-negative is defined as no evidence (RNA or HCV antibodies), past or present.

\section{HPgV-2 Prevalence Study Design}

We used previously described HPgV-2 molecular (11) and serologic (3) assays to test all samples for determining HPgV-2 prevalence. We divided them into 3 testing groups: sample set 1 , initial blood samples ( $\mathrm{n}=402$ ); sample set 2 , all last available follow-up samples ( $n=200)$; and sample set 3 , any longitudinal samples available for samples that were HPgV2 (RNA or antibody) positive at initial or last draw timepoint $(n=70)$ (Figure). Because the initial study collection targeted incident HCV infection, a limited number of participants who were HCV positive at initial collection had follow-up samples available; only $8 \mathrm{HCV}$-positive and $192 \mathrm{HCV}$-negative participants from sample set 1 had follow-up (last) draw available for testing, constituting sample set 2 .

\section{HPgV-2 Molecular Assay}

We used a modified version of the HPgV-2 reverse transcription PCR (RT-PCR) to determine HPgV-2 viremia (11). The RT-PCR targets 2 conserved regions of the $\mathrm{HPgV}$-2 genome within the $5^{\prime}$ untranslated region (UTR) and the nonstructural (NS) 2-3 coding region (11). We modified the assay to replace detection of $\mathrm{HPgV}$ RNA with an internal control. The internal control was derived from the hydroxypyruvate reductase gene from the pumpkin plant, Cucurbita pepo, and is delivered in an Armored RNA (Ambion, Inc., https:/ / www.thermofisher.com) particle that has been diluted in negative human plasma (nonreactive for $\mathrm{HBsAg}$, HIV RNA, HCV RNA, HBV DNA, HIV-1/-2 antibodies, and HCV antibodies). We introduced the internal control into each specimen at the beginning of sample preparation as a control for extraction and amplification. We extracted samples from plasma using

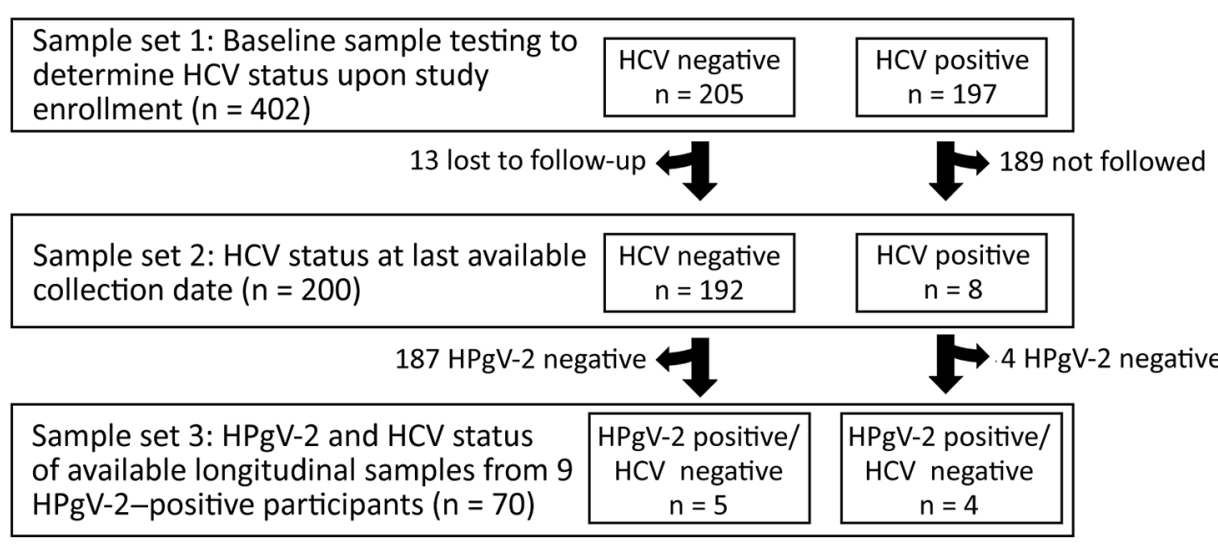

Figure. Design of study of chronic human pegivirus-2 and hepatitis $C$ virus co-infection in injection drug users in the San Francisco Bay area, California, USA. Samples were tested using $\mathrm{HPgV}-2$ molecular and serologic assays in 3 sample sets. HCV, hepatitis $\mathrm{C}$ virus; HPgV-2, human pegivirus 2 . 
the Abbott m2000sp instrument (Abbott Molecular, https://www.molecular.abbott) (open mode protocol m2000-RNA-Plasma-LL-500-110-v71408, version 1.0). We used eluted nucleic acids immediately for subsequent PCR analysis or stored them in the deep well plate at $-80^{\circ} \mathrm{C}$.

\section{HPgV-2 Antibody Testing}

We screened research use-only assays to detect IgG response to $\mathrm{HPgV}-2$ proteins for $\mathrm{HPgV}-2$ seroconversion (3). In brief, we built 2 separate indirect IgG assays for use on the ARCHITECT instrument (Abbott Laboratories). The capture antigen for the E2 assay was mammalian expressed glycoprotein E2, and for the NS4AB assay a portion of the NS4AB region. We generated signal to cutoff values for each assay by determining a provisional cutoff from testing a population of lowrisk volunteer donors and calculating the median +10 SD of relative light units (RLU) generated using the individual assays (3). Both E2 and NS4AB assays detected active and resolved $\mathrm{HPgV}-2$ infection (3).

\section{Statistical Analyses}

We used the Fisher exact test to examine differences in prevalence of $\mathrm{HPgV}-2$ between subgroups (for example, by HCV status). We performed unpaired Student $t$-tests to determine if there was a significant difference $(\mathrm{p}<0.05)$ between the average HPgV-2 log copies/mL (NS2/3 or 5'UTR) of the HCV positive and negative groups. We used GraphPad Prism version 6.04 for Windows (GraphPad Software, https:/ / www.graphpad.com).

\section{Results}

\section{Baseline Sample Testing}

The overall prevalence of $\mathrm{HPgV}-2$ (presence of RNA or antibodies) among baseline samples in the IDU cohort was 6.5\% (Table 1; Figure). We determined a higher $\mathrm{HPgV}-2$ prevalence in the $\mathrm{HCV}$-positive group $(11.2 \%)$ compared with the HCV-negative group $(1.9 \%)(p=0.0002$ by Fisher exact test). We observed $\mathrm{HPgV}-2$ infection (HPgV-2 RNA) more frequently in the HCV-positive group $(6.1 \% ; 12 / 197$ samples) than in the HCV-negative group (1.0\%; 2/197 samples).

\section{Last Sample Testing}

Follow-up specimens were available for some study participants (sample set 2), primarily those who were HCV negative, due to the prospective design of the study that did not require follow-up samples from HCV-positive participants (Figure). However, the study also included persons with newly detected HCV infection whom we followed to examine natural history and resolution of incident HCV infection (9). A total of 200 participants from baseline collection had a final follow-up sample available for evaluation in the HPgV-2 RNA and antibody assays; this group included $8 \mathrm{HCV}$-positive and $192 \mathrm{HCV}$-negative participants (Figure). Although 26 participants were $\mathrm{HPgV}$ 2 positive (by RNA or antibodies) at baseline (Table 1 ), only 4 participants were available for follow-up; they provided $3 \mathrm{HPgV}-2 \mathrm{RNA}$-positive samples and 1 HPgV-2 RNA-negative seropositive sample.

We saw evidence of HPgV-2 infection (antibodies or RNA) in 9 samples in set 2, $6 \mathrm{HCV}$-negative samples and $3 \mathrm{HCV}$-positive samples (Table 2). Among the HCV-negative participants, 3 (QM0003, VH0052, VP0295) showed active HPgV-2 infection during either the baseline or last draw timepoint. Participant QM0003 showed chronic (>6 mos viremia) HPgV-2 infection during the study, with detection of $\mathrm{HPgV}-2$ RNA at time points spanning 832 days (Table 2). Participant VH0052 was actively infected with $\mathrm{HPgV}-2$ at baseline and resolved infection by the last draw date (201 days elapsed), whereas participant VP0295 acquired HPgV-2 infection during the study and was RNA positive at the last draw date (on study for 553 days). Three participants (GG0012, RM0095, RM0337) were $\mathrm{HPgV}-2$ RNA and antibody negative at enrollment and had seroconverted to have HPgV-2 antibodies by the last draw. One participant, VH0044, was $\mathrm{HPgV}-2$ seropositive at baseline but was seronegative (seroreverted) by the final draw ( 250 days elapsed).

In the HCV-positive group, 1 participant (VH0085) was HPgV-2 RNA positive both on the first and last draw dates (2,805 days elapsed). The other $2 \mathrm{HCV}$-positive participants (GG0038 and VT0031) were $\mathrm{HPgV}-2$ RNA negative on the first draw dates but showed active HPgV-2 infection at the last draw date. A single participant (RM0337) acquired both

\begin{tabular}{|c|c|c|c|c|c|c|}
\hline HCV status & No. tested & No. HPgV-2 Ab+ & $\begin{array}{l}\text { No. HPgV-2 } \\
\text { Ab+/RNA+ }\end{array}$ & $\begin{array}{c}\text { No. HPgV-2 } \\
\text { Ab-/RNA+ }\end{array}$ & Total RNA+ & $\begin{array}{c}\text { Total HPgV-2 } \\
\text { RNA or Ab+ }(\%)\end{array}$ \\
\hline HCV positive & 197 & 18 & 8 & 4 & 12 & $22(11.2)$ \\
\hline HCV negative & 205 & 3 & 1 & 1 & 2 & $4(1.9)$ \\
\hline Totals & 402 & 21 & 9 & 5 & 14 & $26(6.5)$ \\
\hline
\end{tabular}

${ }^{*} \mathrm{Ab}$, antibody; $\mathrm{HCV}$, hepatitis $\mathrm{C}$ virus; $\mathrm{HPgV}-2$, human pegivirus 2 ; +, positive; -, negative. 
Table 2. Evidence of HPgV-2 infection in most recent samples from study of chronic HPgV-2 infection and HCV co-infection in injection drug users in the San Francisco Bay area, California, USA*

\begin{tabular}{|c|c|c|c|c|c|c|c|c|}
\hline \multirow{2}{*}{$\begin{array}{l}\text { HCV RNA status } \\
\text { at initial draw }\end{array}$} & \multirow[b]{2}{*}{ Sample ID } & \multicolumn{2}{|c|}{$\begin{array}{l}\text { HPgV-2 status at initial } \\
\text { blood draw }\end{array}$} & \multicolumn{2}{|c|}{$\begin{array}{l}\text { HPgV-2 status at last } \\
\text { blood draw }\end{array}$} & \multirow{2}{*}{$\begin{array}{l}\text { HCV RNA } \\
\text { status }\end{array}$} & \multirow[b]{2}{*}{ No. days $†$} & \multirow[b]{2}{*}{ Comments $\ddagger$} \\
\hline & & RNA & Antibody & RNA & Antibody & & & \\
\hline \multirow[t]{7}{*}{ Negative } & QM0003 & Pos & Neg & Pos & Pos & Neg & 832 & Chronic \\
\hline & VH0052 & Pos & Pos & $\mathrm{Neg}$ & Pos & Neg & 201 & Resolved \\
\hline & VP0295 & $\mathrm{Neg}$ & $\mathrm{Neg}$ & Pos & Pos & Neg & 553 & Active \\
\hline & GG0012 & Neg & Neg & $\mathrm{Neg}$ & Pos & Neg & 689 & Resolved \\
\hline & RM0095 & Neg & Neg & $\mathrm{Neg}$ & Pos & Neg & 201 & Resolved \\
\hline & RM0337§ & Neg & Neg & $\mathrm{Neg}$ & Pos & Pos & 1,680 & Resolved \\
\hline & VH0044 & Neg & Pos & $\mathrm{Neg}$ & $\mathrm{Neg}$ & $\mathrm{Neg}$ & 250 & Resolved \\
\hline \multirow[t]{3}{*}{ Positive } & VH0085 & Pos & Pos & Pos & Pos & Neg & 2,805 & Chronic \\
\hline & GG0038 & $\mathrm{Neg}$ & $\mathrm{Neg}$ & Pos & $\mathrm{Neg}$ & Neg & 461 & Active \\
\hline & VT0031 & Neg & Neg & Pos & Pos & Pos & 818 & Active \\
\hline
\end{tabular}

${ }^{*} \mathrm{HCV}$, hepatitis $\mathrm{C}$ virus; $\mathrm{HPgV}-2$, human pegivirus 2; neg, negative; pos, positive.

tDays elapsed between initial and last blood draw.

†Comments of HPgV-2 status based on initial and last draw testing. Chronic indicates $>6$ months with detectable active viremia. Active indicates viremia at last draw. Resolved indicates no viremia detected at last draw, but antibodies were present.

§Participant RM0337 acquired HCV during the study.

$\mathrm{HPgV}-2$ and HCV during the course of the study, with $\mathrm{HPgV}-2$ infection preceding $\mathrm{HCV}$ infection by 280 days (Table 3 ). Within the last sample set, 6 participants demonstrated $\mathrm{HPgV}-2$ infection after initial collection. Three of the participants showed active $\mathrm{HPgV}-2$ viremia and 3 showed resolved HPgV-2 infection as indicated by detection of antibodies only.

\section{Longitudinal Sample Testing}

We tested longitudinal samples ( $N=70$, sample set 3) from $9 / 10$ participants (Table 2) for $\mathrm{HPgV}-2$ antibodies and RNA (Tables 3, 4; Figure). We reported data available for age, sex, years of injection drug use, HIV status, HCV RNA, HCV antibodies, alanine aminotransferase (HCV-positive only), and HCV drug treatment therapy. We did not follow up with additional HPgV-2 testing on participant VH0044, who was negative for $\mathrm{HPgV}-2$ RNA and antibodies (seroreverted) by last sample date (Table 2). We found chronic HPgV-2 (active viremia $>6$ months) in 5 different IDU participants, 3 male and 2 female; 3 (QM0003, VP0295, RM0095) were HCV negative and 2 (VH0085, GG0038) HCV positive. None of the participants demonstrating chronic $\mathrm{HPgV}-2$ infection or seroconversion had evidence of HIV infection. Among the participants who demonstrated chronic $\mathrm{HPgV}-2$ infection and were $\mathrm{HCV}$ negative, participant QM0003 had a longer ( $>5 \mathrm{y}$ ) history of exposure to injection drug use than the other participants, RM0095 and VP0295 (<5 y). Both participants with active HPgV-2 and HCV co-infection (GG0038 and VH0085) had long histories of injecting exposure ( $>5 \mathrm{y}$ ) and maintained $\mathrm{HPgV}-2$ after HCV infection was resolved.

All chronic $\mathrm{HPgV}-2$ infections demonstrated active viremia despite the presence of $\mathrm{HPgV}-2$ antibodies, with most participants generating an $\operatorname{IgG}$ response to the glycoprotein E2 (Tables 3, 4). E2 antibodies developed in all participants with chronic $\mathrm{HPgV}-2$ samples and observed seroconversion (GG0038, QM0003, VP0295, and RM0095) before the other marker, NS4AB antibody (Tables 3, 4). One chronically infected sample, VH0085, contained antibodies to both E2 and NS4AB, but we did not observe the initial seroconversion. Compared with the other samples in this study, VH0085 had the highest signal to cutoff values for both E2 and NS4AB antibody assays, and the HPgV-2 RNA log copies/mL were higher than in most other samples (Table 3). Some participants (VP0295, RM0095, GG0038) demonstrated seroconversion after several months of initial HPgV-2 RNA detection; these participants were chronically infected with $\mathrm{HPgV}-2$.

The median using the NS2/3 assay was 3.26 $\mathrm{HPgV}-2 \log _{10}$ copies/ $\mathrm{mL}$ for the HCV-negative group and $3.21 \mathrm{HPgV}-2 \log _{10}$ copies/mL for the HCVpositive group; using the $5^{\prime}$ UTR, results were 1.71 $\log _{10}$ copies/mL for the HCV-negative group and $1.60 \log _{10}$ copies/mL for the HCV-positive group (Table 5). HCV co-infection did not appear to influence $\mathrm{HPgV}-2$ viral load; the average value showed no significant difference between the $\mathrm{HCV}$-positive and HCV-negative groups (NS2/3, $\mathrm{p}=0.11 ; 5^{\prime} \mathrm{UTR}$, $\mathrm{p}=0.36$ ). One HCV-positive participant, VH0085, was positive for HPgV-2 and HCV RNA at baseline, received HCV treatment (8 weeks ledipasvir/sofosbuvir), and cleared HCV infection. After clearance of $\mathrm{HCV}$, the participant remained $\mathrm{HPgV}-2$ viremic and went on to establish a chronic HPgV-2 infection that lasted 8 years (2,805 days). Participant GG0038 acquired HPgV-2 infection after spontaneous resolution of HCV infection (RNA negative and HCV antibody positive) and maintained active $\mathrm{HPgV}-2$ infection for $>232$ days. 


\section{Conclusions}

The recently identified human pegivirus $\mathrm{HPgV}-2$ has yet to be linked with any disease in humans. Several groups have shown $\mathrm{HPgV}-2$ infection associated with $\mathrm{HCV}$ co-infection (1,3-6). HPgV-2 is a bloodborne virus, and a higher $\mathrm{HPgV}-2$ prevalence is observed among HCV-positive IDUs $(4,6)$. We decoupled the behavior of injection drug use from HCV status by monitoring HCV-negative or HCV-positive IDUs for $\mathrm{HPgV}-2$ infection (RNA and antibodies). We also observed the enrichment of $\mathrm{HPgV}-2$ infection in HCVpositive IDUs (Table 1), as was reported previously $(4,6)$. We found, through longitudinal surveillance of both HCV-negative participants, that $\mathrm{HPgV}-2$ can establish infection and maintain chronic infection in the absence of HCV. We defined chronic infection as detectable $\mathrm{HPgV}-2$ viremia for $>6$ months in $>3$ timepoints that was not associated with particular symptoms. Of 9 participants with evidence of $\mathrm{HPgV}-2$ infection (by RNA or serology), $2 \mathrm{HCV}$-infected participants demonstrated chronic HPgV-2 infection (Table 3), and 3 participants demonstrated chronic $\mathrm{HPgV}-2$ without evidence of past or present HCV infection (Table 4).

Several limitations can contribute to the underestimation of chronic $\mathrm{HPgV}-2$ infection. We identified HPgV-2 in baseline samples from 12 HCV-positive participants; but because of the study design most HCV-positive participants were not followed through subsequent timepoints. Three of these participants did provide longitudinal samples; 2 participants demonstrated chronic $\mathrm{HPgV}-2$ infection. A second limitation is that participants testing negative for $\mathrm{HPgV}-2$ during the timepoints evaluated may become positive following the last timepoint sampled, if they continue the risk behavior of intravenous drug use. Alternatively, false-positive chronic infections could result from long lapses in sampling, in which

Table 3. Information about participants with HPgV-2 infection with HCV co-infection in study of injection drug users in the San Francisco Bay area, California, USA*

\begin{tabular}{|c|c|c|c|c|c|c|c|c|c|}
\hline Sample ID & $\begin{array}{l}\text { Participant } \\
\text { age, y/sex }\end{array}$ & $\begin{array}{l}\text { No. years } \\
\text { drug use }\end{array}$ & Collection date & $\begin{array}{l}\text { HCV } \\
\text { RNA }\end{array}$ & $\begin{array}{c}\text { HCV } \\
\text { antibody }\end{array}$ & $\begin{array}{l}\mathrm{NS} 2 / 3 \log _{10} \\
\text { copies/mL }\end{array}$ & $\begin{array}{l}5^{\prime} \text { UTR } \log _{10} \\
\text { copies/mL }\end{array}$ & $\begin{array}{l}\text { NS4AB } \\
\text { S/CO } \ddagger\end{array}$ & $\begin{array}{c}\mathrm{E} 2 \\
\mathrm{~S} / \mathrm{CO} \ddagger\end{array}$ \\
\hline \multirow[t]{11}{*}{ RM0337 } & $25.6 / \mathrm{F}$ & 11.1 & 2013 Jul 10 & Neg & $\mathrm{Neg}$ & Neg & Neg & 0.16 & 0.14 \\
\hline & & & 2013 Oct 9 & $\mathrm{Neg}$ & $\mathrm{Neg}$ & $\mathrm{Neg}$ & $\mathrm{Neg}$ & 0.14 & 0.13 \\
\hline & & & 2014 Jan 29 & $\mathrm{Neg}$ & $\mathrm{Neg}$ & $\mathrm{Neg}$ & $\mathrm{Neg}$ & 0.13 & 0.12 \\
\hline & & & 2016 Jul 27 & $\mathrm{Neg}$ & $\mathrm{Neg}$ & $\mathrm{Neg}$ & $\mathrm{Neg}$ & 0.12 & 0.11 \\
\hline & & & 2016 Oct 26 & $\mathrm{Neg}$ & $\mathrm{Neg}$ & $\mathrm{Neg}$ & $\mathrm{Neg}$ & 0.12 & 0.13 \\
\hline & & & 2017 Jan 18 & $\mathrm{Neg}$ & $\mathrm{Neg}$ & $\mathrm{Neg}$ & $\mathrm{Neg}$ & 0.13 & 0.13 \\
\hline & & & 2017 Apr 26 & $\mathrm{Neg}$ & $\mathrm{Neg}$ & 0.96 & 0.42 & 0.13 & 1.46 \\
\hline & & & 2017 Jul 19 & $\mathrm{Neg}$ & $\mathrm{Neg}$ & $\mathrm{Neg}$ & $\mathrm{Neg}$ & 0.09 & 1.34 \\
\hline & & & 2017 Oct 18 & $\mathrm{Neg}$ & $\mathrm{Neg}$ & Neg & Neg & 0.13 & 0.88 \\
\hline & & & 2018 Jan 31 & Pos & $\mathrm{Neg}$ & $\mathrm{Neg}$ & Neg & 0.14 & 1.02 \\
\hline & & & 2018 Feb 14 & Pos & $\mathrm{Neg}$ & $\mathrm{Neg}$ & $\mathrm{Neg}$ & 0.25 & 1.26 \\
\hline \multirow[t]{6}{*}{ VH0085§ } & $22.2 / \mathrm{F}$ & 9.9 & 2010 May 14 & Pos & Pos & 3.22 & 2.3 & 1.38 & 9.37 \\
\hline & & & 2017 Feb 15 & $\mathrm{Neg}$ & Not tested & 3.14 & 2.07 & 2.96 & 12.92 \\
\hline & & & 2017 May 17 & Neg & Not tested & 1.87 & 0.69 & 3.33 & 12.35 \\
\hline & & & 2017 Aug 9 & $\mathrm{Neg}$ & Not tested & 4.30 & 2.25 & 6.13 & 18.71 \\
\hline & & & 2017 Nov 1 & $\mathrm{Neg}$ & Not tested & 3.40 & 1.68 & 5.13 & 19.45 \\
\hline & & & 2018 Jan 17 & $\mathrm{Neg}$ & Not tested & 3.86 & 2.26 & 3.75 & 16.16 \\
\hline \multirow[t]{5}{*}{ GG0038§ } & $26.5 / \mathrm{M}$ & 8.8 & 2016 Jul 14 & Pos & Pos & $\mathrm{Neg}$ & $\mathrm{Neg}$ & 0.16 & 0.12 \\
\hline & & & 2017 Mar 1 & Pos & Not tested & 3.04 & 1.02 & 0.17 & П \\
\hline & & & 2017 May 3 & Pos & Not tested & 2.14 & 0.64 & 0.20 & 0.37 \\
\hline & & & 2017 May 23 & Neg & Not tested & 3.01 & 1.16 & 0.18 & 0.36 \\
\hline & & & 2017 Oct 18 & Neg & Not tested & 4.15 & 1.91 & 0.16 & 2.96 \\
\hline \multirow[t]{10}{*}{ VT0031 } & 19.8/M & 1.8 & 2005 Feb 2 & Pos & Pos & $\mathrm{Neg}$ & $\mathrm{Neg}$ & 0.09 & 0.22 \\
\hline & & & 2005 May 24 & Pos & Pos & $\mathrm{Neg}$ & $\mathrm{Neg}$ & 0.11 & 0.15 \\
\hline & & & 2005 Jun 14 & Pos & Pos & $\mathrm{Neg}$ & $\mathrm{Neg}$ & 0.10 & 0.17 \\
\hline & & & 2005 Aug 9 & Pos & Pos & $\mathrm{Neg}$ & $\mathrm{Neg}$ & 0.12 & 0.14 \\
\hline & & & 2005 Nov 8 & Pos & Pos & $\mathrm{Neg}$ & $\mathrm{Neg}$ & 0.08 & 0.13 \\
\hline & & & 2006 Jan 31 & Pos & Pos & $\mathrm{Neg}$ & $\mathrm{Neg}$ & 0.14 & 0.13 \\
\hline & & & 2006 May 9 & Pos & Pos & $\mathrm{Neg}$ & $\mathrm{Neg}$ & 0.09 & 0.19 \\
\hline & & & 2006 Aug 8 & Pos & Pos & $\mathrm{Neg}$ & $\mathrm{Neg}$ & 0.21 & 1.25 \\
\hline & & & 2007 Feb 6 & Pos & Pos & 2.30 & 0.53 & 0.14 & 1.19 \\
\hline & & & 2007 May 1 & Pos & Pos & 0.48 & 0.41 & 0.14 & 1.82 \\
\hline
\end{tabular}

*Participants were determined positive for HCV by RNA or antibody test. HCV, hepatitis C virus; HPgV-2, human pegivirus 2; ID, identification; neg, negative; NS, nonstructural protein; pos, positive; S/CO, signal to cutoff value; UTR, untranslated region.

$\dagger$ Number of years participant had injected drugs as of the time of the initial blood draw.

$\pm \mathrm{S} / \mathrm{CO}>1.0$ is considered positive.

§Participants VH0085 and GG0038 demonstrate resolved HCV infection. VH0085 was administered direct active antiviral drugs (8 weeks ledipasvir/sofosbuvir, LDV-SOF), and GG0038 spontaneously cleared HCV infection.

TVolume not available for testing. 
the participant could become infected, clear the infection, then become reinfected with $\mathrm{HPgV}-2$. The molecular and serologic assays cannot distinguish reinfection from chronic infection.

We observed $\mathrm{HPgV}-2$ seroconversion in the longitudinal surveillance of 7 participants; the detectable IgG response occurred several months after initial HPgV-2 RNA detection in several of the chronically infected participants (Tables 3, 4). Similar to HCV infection, which demonstrates a seronegative viremic window period of 50-60 days (12-14), the detection of $\mathrm{HPgV}-2$-specific antibodies lagged behind detectable HPgV-2 RNA (Tables $3,4)$. In this study, all participants demonstrating chronic $\mathrm{HPgV}-2$ viremia did so in the presence of antibodies to the glycoprotein E2, which suggests that E2 antibodies are not neutralizing. In contrast, active viremia and
E2 IgG are rarely co-detected in persons with the closest human virus, human pegivirus- $1(\mathrm{HPgV}, \mathrm{GBV}-\mathrm{C})$; the presence of $\mathrm{E} 2$ antibodies in $\mathrm{HPgV}$ infection often indicates resolution of infection $(3,15-17)$.

Our data indicate that chronic $\mathrm{HPgV}-2$ infection among IDUs does not require active $\mathrm{HCV}$ to establish infection or maintain chronic infection. It is possible that participants with no detectable $\mathrm{HCV}$ antibodies were seroreverters from previous cleared $\mathrm{HCV}$ infections; however, this is unlikely in our study because the cohort of active IDUs had ongoing exposure to $\mathrm{HCV}$, and because seroreversion in immunocompetent persons has been shown to occur after a long time (>7 years) (18), exceeding the observation period of this study. Furthermore, 2 participants showed spontaneous (GG0038) or therapeutic (VH0085) resolution of

\begin{tabular}{|c|c|c|c|c|c|c|c|}
\hline & & No. years drug & & NS2/3 $\log _{10}$ & $5^{\prime}$ UTR $\log _{10}$ & NS4AB & \\
\hline \multirow{11}{*}{$\frac{\text { Sample ID }}{\text { QM0003 }}$} & Age, y/sex & use† & Collection date & copies/mL & copies/mL & S/CO & E2 S/CO‡ \\
\hline & $28.4 / F$ & 14.4 & 2015 Oct 10 & 3.76 & 2.84 & 0.12 & 0.08 \\
\hline & & & 2015 Nov 4 & 0.93 & 0.55 & 0.08 & 0.08 \\
\hline & & & 2016 Jan 27 & 2.66 & 1.10 & 0.16 & 0.72 \\
\hline & & & 2016 Apr 18 & 3.56 & 1.62 & 0.07 & 0.85 \\
\hline & & & 2016 Jul 13 & 3.66 & 1.61 & $\S$ & $\S$ \\
\hline & & & 2016 Oct 5 & 3.22 & 1.74 & 0.13 & 2.76 \\
\hline & & & 2017 Jan 4 & 4.22 & 2.30 & 0.25 & 4.67 \\
\hline & & & 2017 Apr 10 & 3.85 & 2.10 & 0.17 & 3.58 \\
\hline & & & 2017 Jul 5 & 4.08 & 2.39 & 0.28 & 3.73 \\
\hline & & & 2018 Feb 7 & 3.41 & 1.34 & 0.37 & 2.66 \\
\hline \multirow[t]{6}{*}{ VP0295 } & $24.6 / \mathrm{M}$ & 3.1 & 2016 Jan 6 & Neg & Neg & 0.10 & 0.12 \\
\hline & & & 2016 Apr 6 & $\mathrm{Neg}$ & $\mathrm{Neg}$ & 0.09 & 0.11 \\
\hline & & & 2016 Aug 24 & $\mathrm{Neg}$ & $\mathrm{Neg}$ & 0.12 & 0.11 \\
\hline & & & 2017 Jan 4 & 4.44 & 2.82 & 0.11 & 0.14 \\
\hline & & & 2017 Apr 12 & 3.19 & 1.06 & 0.09 & 0.89 \\
\hline & & & 2017 Jul 12 & 1.42 & 0.82 & 0.15 & 5.51 \\
\hline \multirow[t]{8}{*}{ GG0012 } & $23.4 / F$ & 3.4 & 2016 Apr 29 & Neg & Neg & 0.23 & 0.52 \\
\hline & & & 2016 Aug 17 & $\mathrm{Neg}$ & $\mathrm{Neg}$ & 1.77 & 3.28 \\
\hline & & & 2016 Nov 29 & Neg & Neg & 3.18 & 5.76 \\
\hline & & & 2017 Mar 1 & Neg & Neg & 4.90 & 8.90 \\
\hline & & & 2017 May 31 & Neg & Neg & 2.60 & 4.51 \\
\hline & & & 2017 Sep 20 & Neg & Neg & 4.05 & 10.94 \\
\hline & & & 2017 Dec 13 & Neg & Neg & 6.03 & 11.04 \\
\hline & & & 2018 Mar 19 & Neg & Neg & 3.77 & 6.67 \\
\hline \multirow[t]{2}{*}{ VH0052 } & 23.7/M & 3.5 & 2006 Dec 14 & 2.13 & 1.23 & 2.00 & 1.91 \\
\hline & & & 2007 Jul 3 & $\mathrm{Neg}$ & $\mathrm{Neg}$ & 0.81 & 1.63 \\
\hline \multirow[t]{12}{*}{ RM0095 } & $27.1 / \mathrm{M}$ & 1.1 & 2011 Jan 19 & $\mathrm{Neg}$ & Neg & 0.06 & 0.06 \\
\hline & & & 2011 Jul 27 & 0.02 & 0.14 & 0.07 & 0.09 \\
\hline & & & 2011 Oct 19 & 2.33 & 1.54 & 0.06 & 0.29 \\
\hline & & & 2012 Jan 11 & 0.20 & 0.50 & 0.09 & 0.59 \\
\hline & & & 2012 Apr 11 & 0.02 & 0.16 & 0.24 & 0.50 \\
\hline & & & 2012 Jul 3 & $\mathrm{Neg}$ & Neg & 0.14 & 0.59 \\
\hline & & & 2012 Oct 23 & 0.03 & 0.27 & 0.12 & 1.09 \\
\hline & & & 2013 Jan 15 & 0.45 & 0.61 & 0.15 & 2.45 \\
\hline & & & 2013 Apr 10 & 1.37 & 1.08 & 0.13 & 2.37 \\
\hline & & & 2013 Jul 2 & Neg & Neg & 0.17 & 3.53 \\
\hline & & & 2013 Sep 18 & 0.46 & 0.55 & 0.14 & 2.64 \\
\hline & & & 2013 Dec 11 & $\mathrm{Neg}$ & Neg & 0.14 & 3.07 \\
\hline
\end{tabular}

*Participants were determined negative for HCV by RNA or antibody test. HCV, hepatitis C virus; HPgV-2, human pegivirus 2; ID, identification; neg, negative; NS, nonstructural protein; pos, positive; S/CO, signal to cutoff value; UTR, untranslated region. †Number of years participant had injected drugs as of the time of the initial blood draw. $\pm \mathrm{S} / \mathrm{CO}>1.0$ is considered positive.

$\S$ No blood sample available for testing. 
Human Pegivirus 2 without Hepatitis C Co-infection

Table 5. Characteristics of findings in study of chronic HPgV-2 infection for participants with and without HCV co-infection in injection drug users in the San Francisco Bay area, California, USA*

\begin{tabular}{|c|c|c|c|}
\hline Finding & HCV negative, $n=3$ & HCV positive, $n=2$ & $p$ value \\
\hline \multicolumn{4}{|l|}{ Average HPgV-2, $\log _{10}$ copies $/ \mathrm{mL}$} \\
\hline NS2/3 & 3.26 & 3.21 & 0.11 \\
\hline 5' UTR & 1.71 & 1.60 & 0.36 \\
\hline Average years injection drug use $\dagger$ & 5.5 & 7.9 & NA \\
\hline Average age at detection of $\mathrm{HPgV}-2$ RNA, y† & 25.6 & 26.1 & NA \\
\hline
\end{tabular}

$\mathrm{HCV}$ infection but HPgV-2 chronic infection remained (Table 3); thus, there appeared to be no reliance on $\mathrm{HCV}$ to establish or maintain $\mathrm{HPgV}-2$ infection. We observed no difference in $\mathrm{HPgV}-2$ viral loads whether $\mathrm{HCV}$ was present or absent (Table 5). The relative ratio of resolved to active infections between the HCV-positive and HCV-negative cohorts was similar (Table 1).

As noted in this and other studies, high-risk populations that are exposed to parenterally transmitted viruses experience an increase in $\mathrm{HPgV}-2$ prevalence $(4,6,7)$. We observed similar higher incidence of both active and resolved $\mathrm{HPgV}-2$ infection in the HCVpositive IDU cohort (Table 1). The HCV-positive and $\mathrm{HCV}$-negative infected persons within the IDU cohort share many common behaviors with no discernable characteristics, except total number of years of injection drug use (average 7.6 y for HCV-positive users, $4.7 \mathrm{y}$ for HCV-negative users). Similarly, the $\mathrm{HCV}$-positive $\mathrm{HPgV}-2$ carriers identified in this study demonstrated injection drug use behavior longer (average $7.9 \mathrm{y}$ ) than the HCV-negative group (average $4.4 \mathrm{y}$ ). The greater number of potential exposures to parentally transmitted bloodborne viruses is probably a major contributing factor for increased prevalence of $\mathrm{HPgV}-2$ in the $\mathrm{HCV}$-positive IDU cohort.

The pathogenic potential of $\mathrm{HPgV}-2$ in humans remains unknown; no clinical symptoms have been associated with $\mathrm{HPgV}-2$ infection. We gathered no additional clinical information from $\mathrm{HCV}$-negative study participants. Most $\mathrm{HPgV}-2$ studies have shown the virus is associated with $\mathrm{HCV}$ co-infection, which can mask any pathogenicity associated with $\mathrm{HPgV}$ 2 infection. Identifying populations that show higher prevalence of HPgV-2 monoinfection and monitoring these persons over time may help identify clinical symptoms associated with $\mathrm{HPgV}-2$ infection, thus enabling researchers to categorize $\mathrm{HPgV}-2$ as human pathogen or benign infection.

\section{Acknowledgments}

We acknowledge the ongoing support and efforts of the Blood Systems Research Institute, especially Michael Busch, Mars Stone, and Honey Dave, for their expert management of the UFO Study specimen bank.

\section{About the Author}

Dr. Coller is a research scientist at Abbott Laboratories, Abbott Diagnostics Division. Her research interests include developing serologic assays for the detection of emerging infectious diseases.

\section{References}

1. Berg MG, Lee D, Coller K, Frankel M, Aronsohn A, Cheng $K$, et al. Discovery of a novel human pegivirus in blood associated with hepatitis C virus co-infection. PLoS Pathog. 2015;11:e1005325. https:// doi.org/10.1371/ journal.ppat.1005325

2. Kapoor A, Kumar A, Simmonds P, Bhuva N, Singh Chauhan L, Lee B, et al. Virome analysis of transfusion recipients reveals a novel human virus that shares genomic features with hepaciviruses and pegiviruses. MBio. 2015;6:e01466-15. https://doi.org/10.1128/mBio.01466-15

3. Coller KE, Berg MG, Frankel M, Forberg K, Surani R, Chiu CY, et al. Antibodies to the novel human pegivirus 2 are associated with active and resolved infections. J Clin Microbiol. 2016;54:2023-30. https:/ / doi.org/10.1128/ JCM.00515-16

4. Wang H, Wan Z, Sun Q, Zhu N, Li T, Ren X, et al. Second human pegivirus in hepatitis $C$ virus-infected and hepatitis $\mathrm{C}$ virus/HIV-1-co-infected persons who inject drugs, China. Emerg Infect Dis. 2018;24:908-11. https:/ / doi.org/10.3201/ eid2405.161162

5. Wang H, Wan Z, Xu R, Guan Y, Zhu N, Li J, et al. A novel human pegivirus, $\mathrm{HPgV}-2(\mathrm{HHpgV}-1)$, is tightly associated with hepatitis $\mathrm{C}$ virus (HCV) infection and $\mathrm{HCV} /$ human Immunodeficiency virus type 1 coinfection. Clin Infect Dis. 2018;66:29-35. https://doi.org/10.1093/cid/cix748

6. Kandathil AJBF, Thomas DL, Balagopal A, Mehta SH, Timp W, Salzberg SL, et al. Presence of human hepegivirus-1 in a cohort of people who inject drugs. Ann Intern Med. 2018;168:158-9. https://doi.org/10.7326/L17-0527

7. Bonsall D, Gregory WF, Ip CLC, Donfield S, Iles J, Ansari MA, et al. Evaluation of viremia frequencies of a novel human pegivirus by using bioinformatic screening and PCR. Emerg Infect Dis. 2016;22:671-8. https:/ / doi.org/ 10.3201/eid2204.151812

8. Bijvand Y, Aghasadeghi MR, Sakhaee F, Pakzad P, Vaziri F, Saraji AA, et al. First detection of human hepegivirus-1 (HHpgV-1) in Iranian patients with hemophilia. Sci Rep. 2018;8:5036. https://doi.org/10.1038/s41598-018-23490-4

9. Page K, Hahn JA, Evans J, Shiboski S, Lum P, Delwart E, et al. Acute hepatitis $C$ virus infection in young adult injection drug users: a prospective study of incident infection, resolution, and reinfection. J Infect Dis. 2009;200:1216-26. https:/ / doi.org/10.1086/605947

10. Hahn JA, Page-Shafer K, Lum PJ, Bourgois P, Stein E, Evans JL, et al. Hepatitis $C$ virus seroconversion among 
young injection drug users: relationships and risks. J Infect Dis. 2002;186:1558-64. https://doi.org/10.1086/345554

11. Frankel M, Forberg K, Coller KE, Berg MG, Hackett J Jr, Cloherty G, et al. Development of a high-throughput multiplexed real time RT-PCR assay for detection of human pegivirus 1 and 2. J Virol Methods. 2017;241:34-40. https:/ / doi.org/10.1016/j.jviromet.2016.12.013

12. Cox AL, Netski DM, Mosbruger T, Sherman SG, Strathdee S, Ompad D, et al. Prospective evaluation of communityacquired acute-phase hepatitis $C$ virus infection. Clin Infect Dis. 2005;40:951-8. https:// doi.org/10.1086/428578

13. Maheshwari A, Ray S, Thuluvath PJ. Acute hepatitis C. Lancet. 2008;372:321-32. https:// doi.org/10.1016/ S0140-6736(08)61116-2

14. Glynn SA, Wright DJ, Kleinman SH, Hirschkorn D, Tu Y, Heldebrant $C$, et al. Dynamics of viremia in early hepatitis $C$ virus infection. Transfusion. 2005;45:994-1002. https://doi.org/10.1111/j.1537-2995.2005.04390.x

15. Tanaka T, Hess G, Schlueter V, Zdunek D, Tanaka S, Kohara M. Correlation of interferon treatment response with GBV-C/HGV genomic RNA and anti-envelope 2 protein antibody. J Med Virol. 1999;57:370-5. https:/ / doi.org/10.1002/ (SICI)1096-9071(199904)57:4<370::AID-JMV8>3.0.CO;2-K

16. Gutierrez RA, Dawson GJ, Knigge MF, Melvin SL, Heynen CA, Kyrk CR, et al. Seroprevalence of GB virus $\mathrm{C}$ and persistence of RNA and antibody. J Med Virol. 1997;53:167-73. https:/ / doi.org/10.1002/(SICI)10969071(199710)53:2<167::AID-JMV10>3.0.CO;2-G

17. Dille BJ, Surowy TK, Gutierrez RA, Coleman PF, Knigge MF, Carrick RJ, et al. An ELISA for detection of antibodies to the E2 protein of GB virus C. J Infect Dis. 1997;175:458-61. https://doi.org/10.1093/infdis/175.2.458

18. Lefrère JJ, Girot R, Lefrère F, Guillaume N, Lerable J, Marrec NL, et al. Complete or partial seroreversion in immunocompetent individuals after self-limited HCV infection: consequences for transfusion. Transfusion. 2004; 44:343-8. https:/ / doi.org/10.1111/j.1537-2995.2004.00656.x

Address for correspondence: Kelly E. Coller, Abbott Laboratories, Abbott Diagnostics Division, 100 Abbott Park Rd, Abbott Park, IL 60064-6400, USA; email: kelly.coller@abbott.com

\section{etymologia}

\section{Pegivirus [peg"i-vi'ros]}

\section{Ronnie Henry}

I n 1967, researchers studying non-A, non-B

Subsequent phylogenetic analysis showed Lhepatitis identified a transmissible agent in the serum of a surgeon (initials G.B.) with acute hepatitis and named it the GB agent. In the 1990s, researchers from Abbott Laboratories identified 3 GB viruses (A, B, and C) at the same time as a group at Genelabs isolated RNA from patients with non-A, non-B hepatitis and named it hepatitis $G$ virus. Later research showed that $G B$ virus $\mathrm{C}$ and hepatitis $\mathrm{G}$ virus were the same species.

\section{Sources}

1. Linnen J, Wages J Jr, Zhang-Keck ZY, Fry KE, Krawczynski KZ, Alter H, et al. Molecular cloning and disease association of hepatitis $G$ virus: a transfusion-transmissible agent. Science. 1996;271: 505-8. https://doi.org/10.1126/science.271.5248.505

2. Simons JN, Leary TP, Dawson GJ, Pilot-Matias TJ, Muerhoff AS, Schlauder GG, et al. Isolation of novel virus-like sequences associated with human hepatitis. Nat Med. 1995;1:564-9. https://doi.org/ $10.1038 / \mathrm{nm} 0695-564$ that GB viruses $A$ and $C$ (and GB virus D, later identified in bats) should be classified under a new genus, Pegivirus (because they cause persistent infection and because of the historic association with hepatitis $\underline{G}$ ), and GB virus B should be classified as a second species (with hepatitis $\mathrm{C}$ virus) in the genus Hepacivirus. As of 2016, 11 species of Pegivirus had been identified (Pegivirus A-K).

3. Smith DB, Becher P, Bukh J, Gould EA, Meyers G, Monath T, et al. Proposed update to the taxonomy of the genera Hepacivirus and Pegivirus within the Flaviviridae family. J Gen Virol. 2016;97:2894-907. https:/ / doi.org/10.1099/jgv.0.000612

4. Stapleton JT, Foung S, Muerhoff AS, Bukh J, Simmonds P. The GB viruses: a review and proposed classification of GBV-A, GBV-C (HGV), and GBV-D in genus Pegivirus within the family Flaviviridae. J Gen Virol. 2011;92:233-46. https:/ / doi.org/10.1099/vir.0.027490-0

Address for correspondence: Ronnie Henry, Centers for Disease Control and Prevention, 1600 Clifton Rd NE, Mailstop E28, Atlanta, GA 30333, USA; email: boq3@cdc.gov 